\title{
A Rotation-Invariant Transform for Target Detection in SAR Images
}

\author{
Wenxing Ye, Christopher Paulson, Dapeng Oliver Wu, and Jian Li \\ Department of Electrical and Computer Engineering, \\ University of Florida, Gainesville, FL 32611
}

\begin{abstract}
Rotation of targets poses a great challenge for the design of an automatic image-based target detection system. In this paper, we propose a target detection algorithm that is robust to rotation of targets. Our key idea is to use rotation invariant features as the input for the classifier. For an image in Radon transform space, namely $R(b, \theta)$, taking the magnitude of 1-D Fourier transform on $\theta$, we get $\left|F_{\theta}\{R(b, \theta)\}\right|$. The rotation invariance of the coefficients of the combined Radon and 1-D Fourier transform, $\left|F_{\theta}\{R(b, \theta)\}\right|$ was proved in this paper. These coefficients are used as the input to a maximum-margin classifier based on I-RELIEF feature weighting technique. The objective of the I-RELIEF technique is to maximize the margin between two classes and improve the robustness of the classifier against uncertainties. For each pixel of the Synthetic Aperture Radar (SAR) image, a feature vector can be extracted from a sub image centered at that pixel. Then our maximum-margin classifier decides whether the pixel is target or non-target which produces a binary-valued image. We further improved the detection performance by connectivity analysis, image differencing, and diversity combining. Our performance evaluation of the proposed algorithm was based on the data set collected by Swedish CARABAS-II systems. In conclusion, the experimental results show that our proposed algorithm achieved superior performance over the benchmark algorithm.
\end{abstract}

Keywords: VHF SAR, rotation invariant transform, target detection, I-RELIEF

\section{INTRODUCTION}

SAR imaging sensors can provide images of a wide ground region and has the ability to visualize what is being covered by the foliage [1][2]. At the low VHF-band, around $20 \mathrm{MHz}-90 \mathrm{MHz}$, radar waves are more likely to detect targets that exceed a certain dimension. Since this dimension is usually much larger than the leaves and branches, the sensors are able to detect the concealed objects underneath the forest. The reflected radar waves from the hidden objects are used to form SAR images in which the larger targets are seen as brighter areas than the smaller objects.

Another problem is to develop an algorithm that can automatically analyze the image and provide the essential information. For example, the essential information can be scene type, existence of certain objects, or location of all the specified objects. In this paper, all the research is made based on a data set captured by CARABAS-II radar which can be downloaded at [3] for free. The purpose of this project is to locate all the vehicles concealed in the forest which is known as automatic target detection (ATD). Also a benchmark algorithm has been provided with the dataset by [1], but this algorithm detected too many false alarms; therefore, further research needs to be conducted to develop a more accurate algorithm.

Techniques using adaptive boosting [4], extended fractal feature [5], genetic programming [6], multiscale autoregressive (MAR), multiscale autoregressive moving average (MARMA) models, singular value decomposition (SVD) methods [7], and constant false alarm rate (CFAR) processing [8] were studied. According to Lundberg et al. [1], the main technical challenge in designing an ATD algorithm for a forest covered region is not detecting targets, but reducing the false alarm rate to a useful level. The SAR is considered to be a good sensor in the foliage penetrating scenario; however, when the targets are concealed by the forest, the branches and leaves will cause a significant amount of noise to appear in the image. One thing to consider is the density of the forest and

Correspondence author: Prof. Dapeng Oliver Wu, E-mail: wu@ece.ufl.edu, http://www.wu.ece.ufl.edu 
the noise in the image are directly proportional. Another key point is most algorithms work well for open areas, but not in the forest because of the strong noise which is produced by the leaves and branches of the trees.

One important assumption that is made when analyzing the images is that the background clutter is stationary and targets are non-stationary. Now due to this assumption that was made, the target detection problem is equivalent to the change analysis which can be defined as finding the differences between the test image and a reference image. The next important clarification is to define test image as an image in which the algorithm tries to locate the targets from the surrounding area. Also the reference image is an image of the same location as the test image, but taken when the targets have moved to a different location. In order to detect the moving targets in the test image, the algorithm takes the difference of the two photos and the outcome consist mostly of the moving targets. The effectiveness of the change-based ATD scheme has been proved by [9][10][11][12].

Along with the dataset [3], a benchmark algorithm and the results are given in [1]. This algorithm is a statistical hypothesis test followed by a CFAR filter and morphological post processing. In the statistical hypothesis test, the targets are assumed to be deterministic signal while the background clutter and noise are assumed to be Gaussian random variables. Then the decision is made for every pixel in the image according to Neyman-Pearson criterion. Now the statistical hypothesis test is used in the benchmark algorithm which is not the optimal solution because the statistical hypothesis test treats each pixel in the SAR image as independent random variable; therefore, loses the spatial information which is vital for detecting targets in the SAR image. Another reason why the statistical hypothesis test is not optimal is because this test uses the same statistical model for different targets.

In our previous work [13], we used a fundamentally different ATD algorithm which shows improvement in the results. Our past algorithm shares the same change analysis idea with the benchmark algorithm, but our scheme is able to determine the targets from local features of labeled SAR images. Now the previous algorithm leads to a more dedicated classifier for the particular target; however, our algorithm trades generality for performance.

Our framework for the new algorithm is similar to the previous algorithm [13] with the exception of a more advanced local descriptor. The function of the local descriptor is to extract local features from the given region of interest in the image. Then the local features computed for region of interest have been proved to be successful in applications of imagery data analysis [14]. In our previous work [13], local features are vectors whose elements are intensity values extracted from a sliding window centered at a pixel of interest. However, in this paper, an extra rotation invariant transform step is applied to the region of interest. In order to receive the feature vectors which are invariant to different object orientations, we use the outcome of the extra rotation invariant transform as elements of the feature vectors. The diagram of the new algorithm is shown in Fig. 1.

Since all the learning and testing images are considered geometrically registered; therefore, the differencing step is taking the difference of each pixel between the two images. After the differencing step comes the preprocessing step which is a denoising step that removes the obvious background. One of the main benefits from the preprocessing step is that it will be able to improve the performance of the algorithm and the converging speed at the learning stage. Next, the proof of the rotation invariant transform step is introduced in Section 3.1. Now the feature extraction is a step that translates a matrix into a vector which shares the same elements. Following feature extraction comes I-RELIEF or Iterative-RELIEF step which is a feature weighting algorithm looking for a weight vector that maximize the margin between two classes and minimize the margin of elements within the same class. Then the classifier is based on the ratio of the distances from the unknown feature of the two classes. Lastly, the post processing works to cluster nearby pixels, remove small detections and the output is the location of every detection. Refer to later part of this paper and our previous work [13] for more details.

The rest of this paper is organized as below. Section 2 briefly describes the data set. In Section 3, we present our proposed scheme. Section 4 shows the experimental results. Section 5 concludes the paper.

\section{DATA DESCRIPTION}

The dataset collected during a flight campaign held in Sweden in summer 2002 was used for the evaluation of the performance of the algorithm. All the images in the dataset were taken by CARABAS-II ultra-wide-band SAR system mounted on a Sabreliner airplane. This system was operated in the frequency range of $20 \mathrm{MHz}-$ 


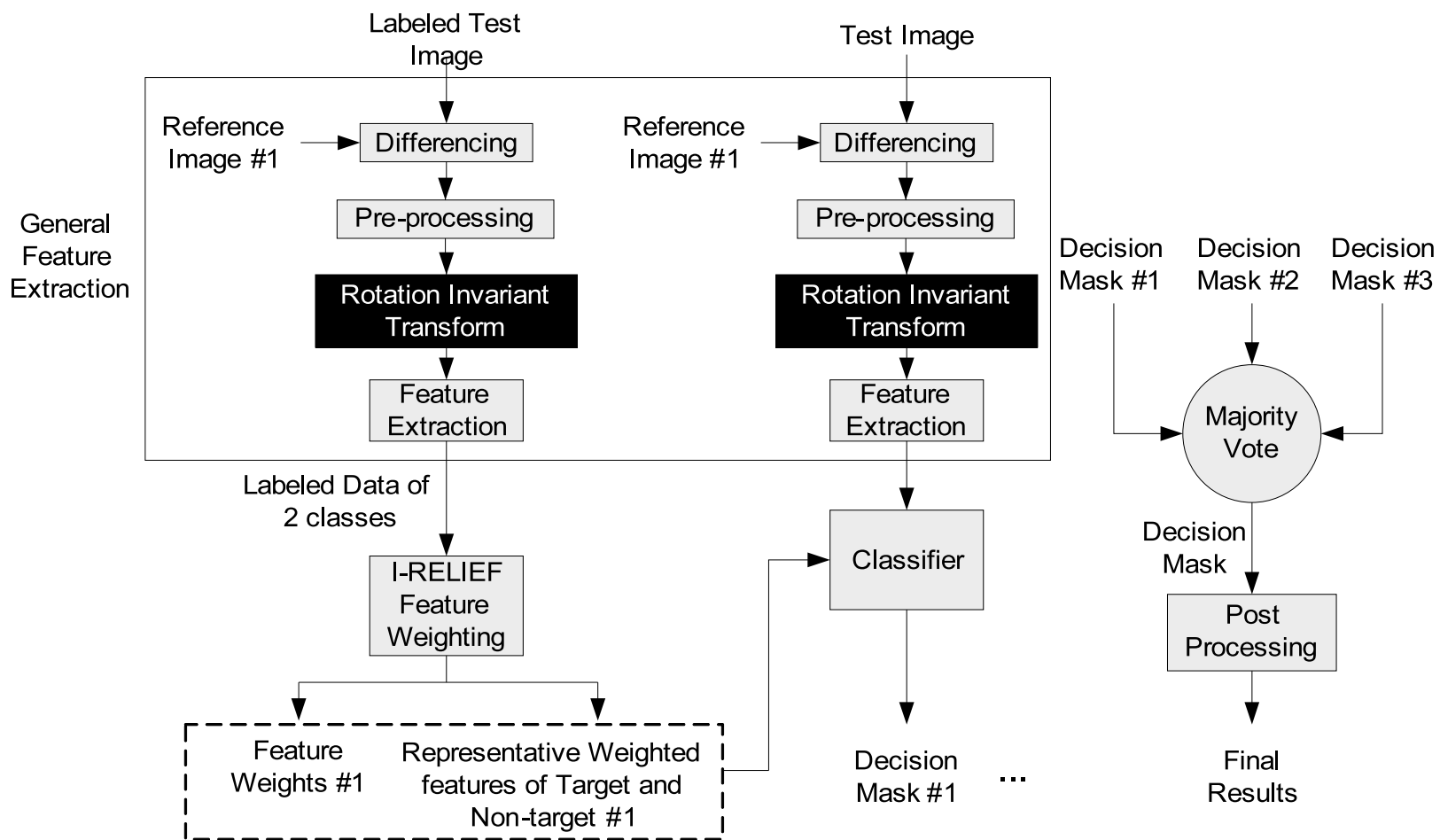

(a)

(b)

(c)

Figure 1: Flow chart of our scheme: (a) learning; (b) testing; (c) diversity combining; black blocks are additional parts in this paper.

$90 \mathrm{MHz}$ which corresponds to the wavelength of 3.3 meters to 15 meters. The wavelengths of 3.3 meters to 15 meters are comparable to the size of vehicles as targets to be pursued.

In this dataset, all the images are $3000 \times 2000$ pixels which are highly accurate intensity matrices that cover the same $3 \mathrm{~km}$ by $2 \mathrm{~km}$ ground area; therefore, the resolution of the data is 1 meter per pixel. There are 24 images in the dataset which were taken at 4 different target locations and 6 flights for each location. For each image, the locations and heading of every vehicle, flight heading, incidence angle, and Radio Frequency Interference (RFI) level are given with the dataset. Fig. 2 shows two sample images and the amplified target regions. Please refer to [1] for more details about the dataset.

\section{PROPOSED TARGET DETECTION SCHEME}

Fig. 1 shows the flow chart of our proposed scheme. It consists of three parts: learning, testing and majority voting.

Learning and testing share the same procedures of differencing, preprocessing, rotation invariant transform and feature extraction. These four common steps can be noted as general feature extraction shown as Fig. 3. This function module serves to extract a feature vector set $\left\{\mathbf{x}_{i}, i=1,2, \ldots, N\right\}$ from the test image $I_{t}(x, y)$ and the reference image $I_{r}(x, y)$. For a given location $(x, y)$ in the image, there would be a corresponding feature vector extracted from a small window centered at $(x, y)$. The small window slides across the image to extract feature vectors from different locations into the output feature vector set. Now to discuss in more detail, the differencing step takes the pixels from the test image and subtracts the pixels from the reference image to remove background noise and clutter. To further suppress the noise in the difference image, both the low pass filter and small threshold are used in this process. First a small threshold is applied to the filtered image in order to remove pixels that are obviously noise. Then the uniform matrix $h$ is used as the convolution kernel of the low pass filter. The calculation of image $I_{d}$ is shown in equation (1). In the third step, the rotation invariant 

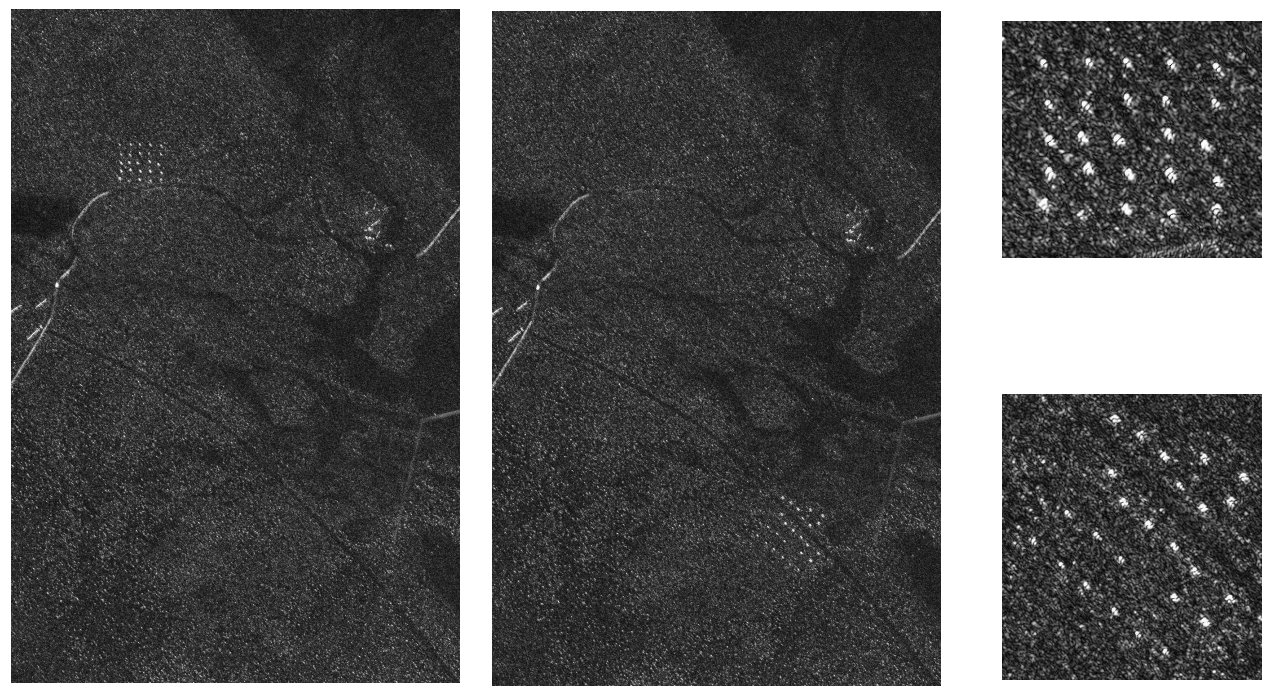

Figure 2: Sample images from dataset. Left: Sigismund deployment, flight heading 225; Middle: Fredrik deployment, flight heading 225; Right: Amplified target regions (25 targets each)

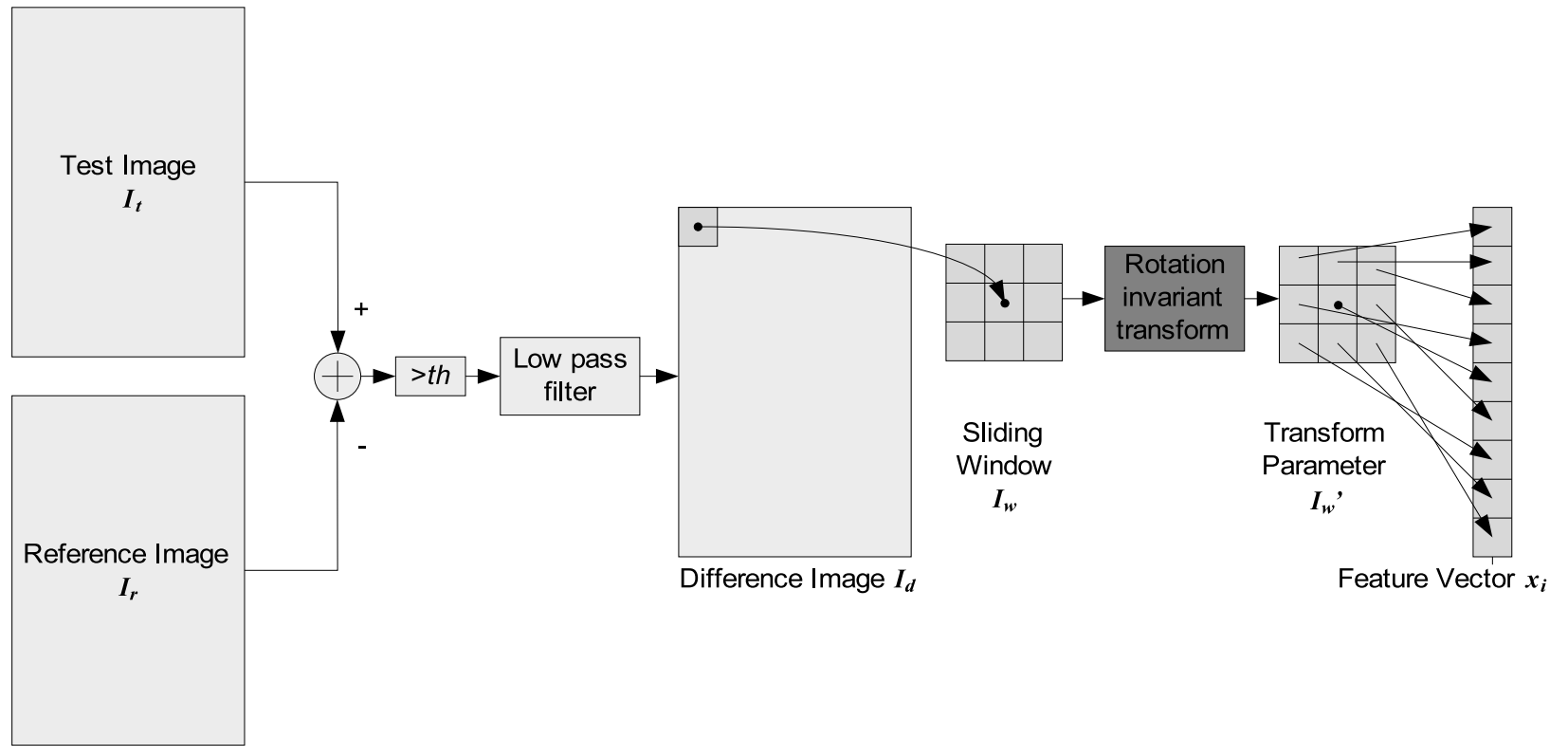

Figure 3: General feature extraction procedure.

transform which will be discussed in section 3.1 was applied. Finally, the feature extraction step is an element reordering step which puts elements of a matrix into a vector in a specific order.

$$
I_{d}= \begin{cases}h *\left(I_{t}-I_{r}\right) & h *\left(I_{t}-I_{r}\right)>t h \\ 0 & h *\left(I_{t}-I_{r}\right) \leq t h\end{cases}
$$

In the learning stage, locations of all the targets are assumed to be given, so the extracted feature set can be labeled as either "1", target or " 0 ", non-target. The labeled feature set is fed to the I-RELIEF feature weighting algorithm to find the best weight vector $\mathbf{w}$ that maximize the margin between two classes of features and minimize the margin within the same class. At the end of this stage, a trained weight vector $\mathbf{w}^{*}$ and two 


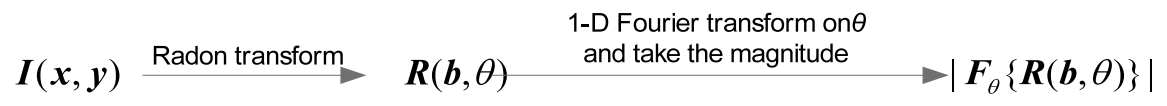

Figure 4: Rotation invariant transform procedure.

representative feature vectors from target and non-target classes are stored for future use in the testing stage. Here, the arithmetic average of all the feature vectors within each class is used as a representative feature vector of that class.

For the testing stage, feature vectors are extracted by moving a sliding window across every possible pixel in the image. Then the vectors are fed to a classifier which is explained in section 3.3. The output of the classifier is then assigned to the corresponding pixel as a decision of " 1 " target or " 0 " non-target. At the end of this stage, a decision mask of a binary valued image is exported to the next step.

Finally, the last stage is comprised of majority voting and post processing. Now majority voting independently makes decisions based on several different reference images and chooses the most frequent output as the final decision. Then the purpose of post processing is to connect all the adjacent target pixels into clusters of potential targets, remove those clusters which are too small to be a target, and output the center of each cluster as the location of detections.

There is an algorithm performance evaluation module after all the above steps to compare the location of every detection given by the algorithm with the ground truth locations from the dataset. If an output location is within certain distance range from any ground truth location, a correct detection is claimed, otherwise, a false alarm has occurred. For each ground truth target, only one correct detection can be assigned. Otherwise the detection rate will be inaccurate because the algorithm may claim more detection than what was actually detected.

\subsection{Rotation Invariant Transform}

Local photometric descriptors computed from interest regions such as Scale Invariant Feature Transform (SIFT) [15] have been used in many applications with great success. However, the manipulated local descriptors are not suitable choices in our research because the target is too small and the image is extremely noisy. In our previous work [13], raw pixel values from a small window are extracted as local features which is the simplest descriptor and indicates good performance of detecting targets.

Raw pixel value descriptor is simple to process and it preserves all the information within the interest region; however, the raw pixel value is a low level local feature which contains a significant amount of redundant information. For example, the same target with different orientations could lead to very different features because the redundant information can easily lead to over fitting or the algorithm diverges in the learning stage if the targets of different orientations were used as training samples.

In our research the detection algorithm was designed to locate targets in SAR images no matter the rotational position of the target. The rotation invariant transform extracts underlying features which is irrelevant to the orientation of the object and use these features to describe the characteristics of the target. Now the extracted rotation invariant feature is an abstraction of the raw data at a higher level which is only related to the target itself but not its orientation. Then, the detection problem is brought into a normalized framework.

Our paper uses an algorithm which consists of Radon transform and Fourier transform shown in Fig. 4. Two dimensional Radon transform is the projection of the image intensity along a radial line oriented at a given angle [16]. A straight line $A A^{\prime}$ in Fig. 5 can be defined parametrically by:

$$
\left\{\begin{array}{l}
x(t)=b \cos \theta+t \sin \theta \\
y(t)=b \sin \theta-t \cos \theta
\end{array}\right.
$$




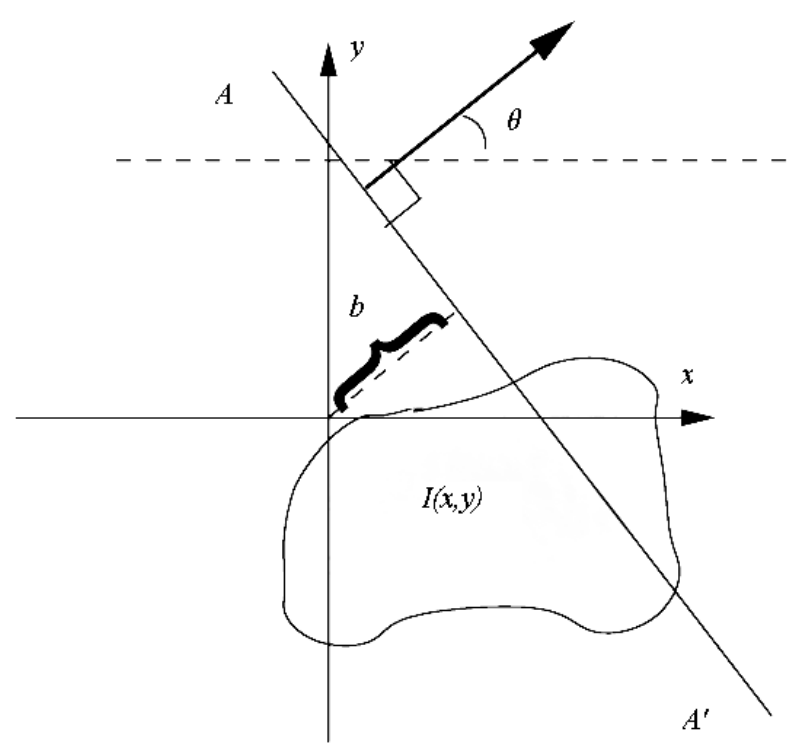

Figure 5: 2-D Radon transform sketch.

Then, the Radon transform can be written as:

$$
R(b, \theta)=\int_{-\infty}^{\infty} I(x(t), y(t)) d t
$$

or the identical expression:

$$
R(b, \theta)=\int_{-\infty}^{\infty} \int_{-\infty}^{\infty} I(x, y) \delta(b-x \cos \theta-y \sin \theta) d x d y
$$

By doing this for different values of $b$ and $\theta$ from 0 degree to 180 degree, the original image $I(x, y)$ is transformed into $R(b, \theta)$.

In order to prove that the proposed rotation invariant transform will get the same output for an original image $I(x, y)$ and its rotated version $I^{\prime}(x, y)$, we will need to prove:

$$
\left|\mathcal{F}_{\theta}\left\{\mathcal{R}\left\{I^{\prime}(x, y)\right\}\right\}=\right| \mathcal{F}_{\theta}\{\mathcal{R}\{I(x, y)\}\}
$$

where $\mathcal{F}_{\theta}\{\bullet\}$ is Fourier transform along the direction of $\theta$ and $\mathcal{R}\{\bullet\}$ is the Radon transform. According to the geometry knowledge, an image rotated by $\theta_{0}$ degree counterclockwise becomes:

$$
I^{\prime}(x, y)=I\left(x \cos \theta_{0}-y \sin \theta_{0}, x \sin \theta_{0}+y \cos \theta_{0}\right)
$$

Then, its Radon transform can be written as:

$$
\begin{aligned}
R^{\prime}(b, \theta) & =\mathcal{R}\left\{I^{\prime}(x, y)\right\}=\int_{-\infty}^{\infty} \int_{-\infty}^{\infty} I^{\prime}(x, y) \delta(b-x \cos \theta-y \sin \theta) d x d y \\
& =\int_{-\infty}^{\infty} \int_{-\infty}^{\infty} I\left(x \cos \theta_{0}-y \sin \theta_{0}, x \sin \theta_{0}+y \cos \theta_{0}\right) \delta(b-x \cos \theta-y \sin \theta) d x d y
\end{aligned}
$$

Define variables $m$ and $n$ as:

$$
\left\{\begin{array}{l}
m=x \cos \theta_{0}-y \sin \theta_{0} \\
n=x \sin \theta_{0}+y \cos \theta_{0}
\end{array}\right.
$$


Then,

$$
\begin{gathered}
\left\{\begin{array}{l}
x=m \cos \theta_{0}+n \sin \theta_{0} \\
y=-m \sin \theta_{0}+n \cos \theta_{0}
\end{array}\right. \\
d x d y=|J| d m d n,|J|=\operatorname{det}\left[\begin{array}{cc}
\cos \theta_{0} & \sin \theta_{0} \\
-\sin \theta_{0} & \cos \theta_{0}
\end{array}\right]=1
\end{gathered}
$$

Pluging equation (9) and (10) into (7) will get:

$$
R^{\prime}(b, \theta)=\int_{-\infty}^{\infty} \int_{-\infty}^{\infty} I(m, n) \delta\left(b-m \cos \left(\theta+\theta_{0}\right)-n \sin \left(\theta+\theta_{0}\right)\right) d m d n=R\left(b, \theta+\theta_{0}\right)
$$

Take the Fourier transform on both sides and take the magnitude, it becomes:

$$
\left|\mathcal{F}_{\theta}\left\{R^{\prime}(b, \theta)\right\}\right|=\left|\mathcal{F}_{\theta}\left\{R\left(b, \theta+\theta_{0}\right)\right\}\right|=\left|e^{j \omega \theta_{0}} \mathcal{F}_{\theta}\{R(b, \theta)\}\right|=\left|\mathcal{F}_{\theta}\{R(b, \theta)\}\right|
$$

Then the proof is done. In the same way, it can be proved that for a rotated and translated image $I^{\prime \prime}(x, y)$ :

$$
\left|\mathcal{F}_{\theta}\left\{\left|\mathcal{F}_{b}\left\{\mathcal{R}\left\{I^{\prime \prime}(x, y)\right\}\right\}\right|\right\}\right|=\left|\mathcal{F}_{\theta}\left\{\left|\mathcal{F}_{b}\{\mathcal{R}\{I(x, y)\}\}\right|\right\}\right|
$$

This is actually a rotation and translation invariant transform. The proof is given but it is not used because the sliding window mechanism which covers every possible translational position is very robust to translational variance and another operation of taking magnitude means more information loss.

Assume $I^{\prime \prime}(x, y)$ is another version of $I(x, y)$ with $\theta_{0}$ degrees counterclockwise rotation followed by $\left(x_{0}, y_{0}\right)$ translation. Then it can be written as:

$$
I^{\prime \prime}(x, y)=I\left(x \cos \theta_{0}-y \sin \theta_{0}+x_{0}, x \sin \theta_{0}+y \cos \theta_{0}+y_{0}\right)
$$

Its Radon transform is:

$$
R^{\prime \prime}(b, \theta)=\int_{-\infty}^{\infty} \int_{-\infty}^{\infty} I\left(x \cos \theta_{0}-y \sin \theta_{0}+x_{0}, x \sin \theta_{0}+y \cos \theta_{0}+y_{0}\right) \delta(b-x \cos \theta-y \sin \theta) d x d y
$$

Denote:

$$
\left\{\begin{array}{l}
m=x \cos \theta_{0}-y \sin \theta_{0}+x_{0} \\
n=x \sin \theta_{0}+y \cos \theta_{0}+y_{0}
\end{array}\right.
$$

Equation (15) turns to be:

$$
\begin{aligned}
R^{\prime \prime}(b, \theta) & =\int_{-\infty}^{\infty} \int_{-\infty}^{\infty} I(m, n) \delta\left(b+x_{0} \cos \left(\theta+\theta_{0}\right)+y_{0} \sin \left(\theta+\theta_{0}\right)-m \cos \left(\theta+\theta_{0}\right)-n \sin \left(\theta+\theta_{0}\right)\right) d m d n \\
& =R\left(b+x_{0} \cos \left(\theta+\theta_{0}\right)+y_{0} \sin \left(\theta+\theta_{0}\right), \theta+\theta_{0}\right)
\end{aligned}
$$

Take the Fourier transform along the direction of $b$ on both sides of equation (17):

$$
\mathcal{F}_{b}\left\{R^{\prime \prime}(b, \theta)\right\}=e^{x_{0} \cos \left(\theta+\theta_{0}\right)+y_{0} \sin \left(\theta+\theta_{0}\right)} \mathcal{F}_{b}\left\{R\left(b, \theta+\theta_{0}\right)\right\}
$$

And:

$$
\left|\mathcal{F}_{b}\left\{R^{\prime \prime}(b, \theta)\right\}\right|=\left|\mathcal{F}_{b}\left\{R\left(b, \theta+\theta_{0}\right)\right\}\right|
$$

Then, further proof of equation (13) is to take the Fourier transform along $\theta$ direction on both sides of equation (19) and take the magnitude.

In this research we used the rotation invariant features instead of the raw pixel values used in our previous work [13] because the rotation invariant algorithm can detect targets of different orientations whereas raw pixel values algorithm is sensitive to change of orientation. Having the capability of being insensitive to different orientations will alleviate the potential of over fitting problem with raw pixel value features. However, the proposed rotation invariant transform needs to take the magnitude of the Fourier transform coefficients which loses all the phase information, so the new feature is less accurate than the raw feature. When using the rotation invariant features the accuracy is traded for generality. 


\subsection{I-RELIEF}

Feature weighting transforms the original feature vector $\mathbf{x}$ into a new feature vector $\mathbf{x}^{\prime}$ by assigning each feature a positive weight $w^{(i)}$. The feature and weight vectors can be defined as:

$$
\mathbf{x}=\left[\begin{array}{c}
x^{(1)} \\
x^{(2)} \\
\vdots \\
x^{(I)}
\end{array}\right] \mathbf{w}=\left[\begin{array}{c}
w^{(1)} \\
w^{(2)} \\
\vdots \\
w^{(I)}
\end{array}\right] \mathbf{x}^{\prime}=\left[\begin{array}{c}
x^{\prime(1)} \\
x^{(2)} \\
\vdots \\
x^{\prime(I)}
\end{array}\right]
$$

where $x^{(i)}=x^{(i)} w^{(i)}, i=1,2, \ldots, I, I$ is the data dimensionality.

I-RELIEF or Iterative-RELIEF is an improved version of RELIEF which is a feature weighting algorithm for increasing the discrimination between classes. The key idea of RELIEF is to solve a convex optimization problem with a margin-based objective function:

$$
\begin{gathered}
\max _{\mathbf{w}} \sum_{n=1}^{N}\left(\sum_{i=1}^{I} w^{(i)}\left|x_{n}^{(i)}-N M^{(i)}\left(x_{n}\right)\right|-\right. \\
\left.\sum_{i=1}^{I} w^{(i)}\left|x_{n}^{(i)}-N H^{(i)}\left(x_{n}\right)\right|\right) \\
\text { s.t. }\|\mathbf{w}\|_{2}^{2}=1, \mathbf{w} \geq 0
\end{gathered}
$$

$N M$ means the nearest miss of $\mathbf{x}$ and $N H$ means the nearest hit of $\mathbf{x}$. Two problems with RELIEF are that $N M$ and $N H$ are defined in the original feature space and the outliers can dramatically influence the margin calculation. To solve the two problems, I-RELIEF calculates the margin based on the probabilities of $N M, N H$, and outliers estimated in the weighted feature space and updates the weights iteratively. Refer to [17] for details.

\subsection{Classifier}

The structure of our classifier is shown in Fig. 6 which is the same as our previous work [13]. Also, our classifier shares the similar philosophy with Neyman-Pearson detector. Now looking at Fig. $6, D_{1}$ and $D_{0}$ are all Euclidean distances and different values of threshold causes a trade off between detection rate and false alarm rate.

\section{EXPERIMENTAL RESULTS}

To evaluate the performance of our algorithm, we set up experiments based on the 24 public SAR images included in the CARABAS-II radar data set. These images are categorized as mission 2, 3, 4 and 5 according to the 4 different vehicle locations. For each location, 6 images known as pass 1 through 6 , were taken in different operating conditions such as flight heading, incidence angle, and radio frequency interference level. Once we receive a test image we always chose the reference image from the different locations but under the same operating conditions; therefore, for any test image, there could be 3 independent reference images for three independent training processes.

The image of mission 3 pass 5 was always used as the test image in the learning stage from which target and non-target training sets were extracted. Now the I-Relief feature weighting algorithm will generate an optimized weight vector $\mathbf{w}^{*}$ from the two training sets. We put the $\mathbf{w}^{*}$ weight vector together with the averages of the two sets of training samples into our classifier. The above procedures were repeated for each reference image to finish the learning stage. Finally, we obtained three classifiers for the three reference images with the same structure but different parameters.

In the testing stage for each test and reference image pair, the classifier trained in the learning stage will make a decision for each pixel and output a decision mask. Since there are three independent reference images there will have three independent decision masks which by majority voting will merge into one final decision mask. Then post processing of clustering will apply to the mask and the centroid of a large enough cluster will 


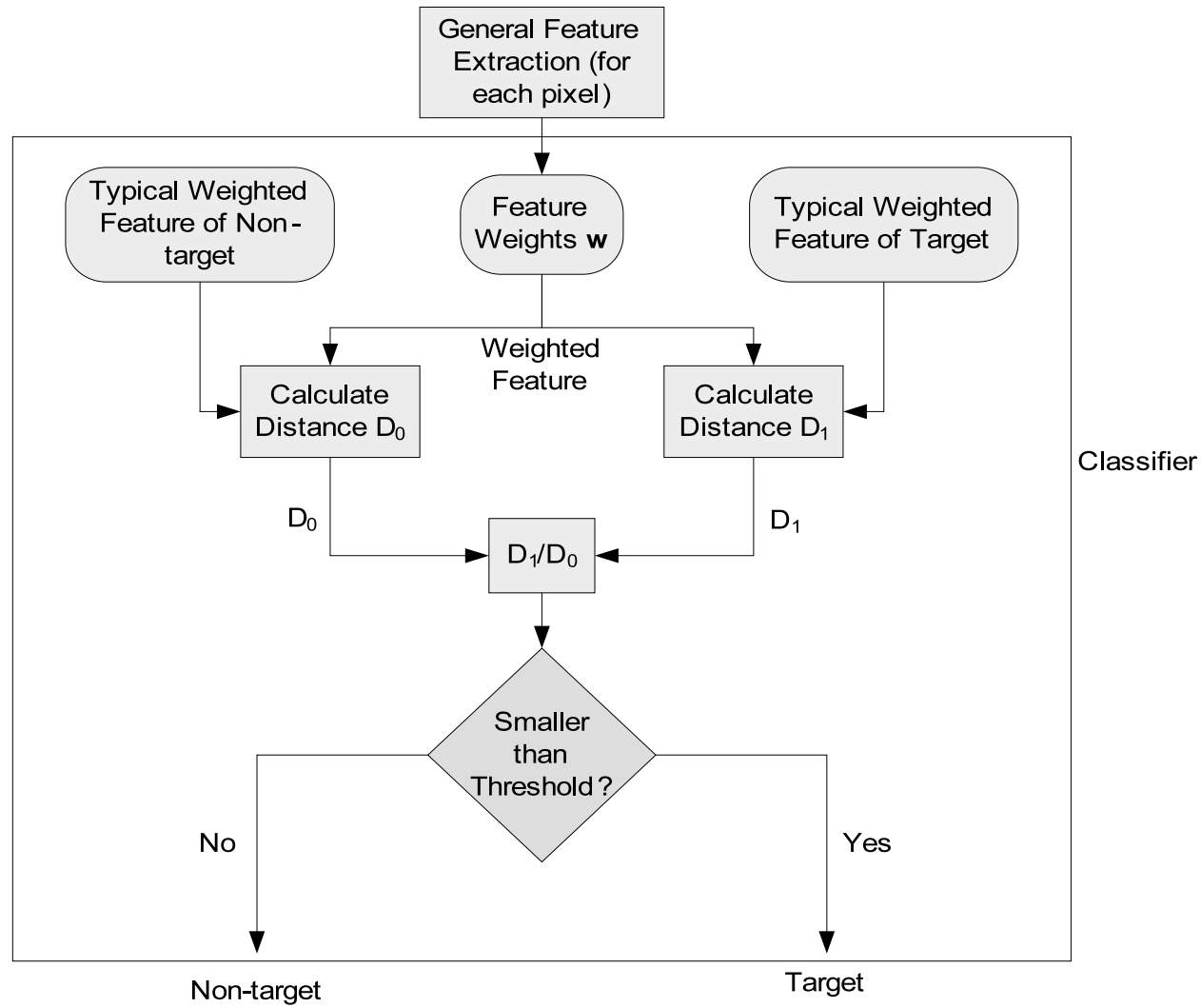

Figure 6: The diagram of classification.

mark the position of the detection. When evaluating the performance of our algorithm, we compare the position of the detections with the locations of real targets and if the distance is less than 10 pixels (i.e. 10 meters), we claim that one detection has been made. For each real target location, only one detection can be claimed and the rest of our detections are false alarms.

The above experiment setup is the same as our previous work [13], except an additional rotation invariant module was added to the experiment which is implemented with the help of Radon Transform and Fast Fourier Transform (FFT). Actually Radon Transform requires discrete values of $\theta$ between $0^{\circ}$ and $180^{\circ}$ as inputs. In our experiment, $\theta$ is chosen to be $0^{\circ}, 15^{\circ}, 30^{\circ}, 45^{\circ}, \ldots, 180^{\circ}$. When the Radon Transform is applied to the sliding window of $19 \times 19$ with the given $\theta$ values, the feature vector increases its dimension from 361 to 377 . Since the output of the Radon transform is a $29 \times 13$ instead of $19 \times 19$ matrix and FFT does not change its dimension.

Table 1 shows the parameters used in our experiment. Table 2 shows the experimental results from our algorithm compared with the benchmark algorithm. However, image of mission 3 pass 5 was not used for testing because it served as a training set. In conclusion, our experimental results show that our algorithm produces a lower false alarm rate and higher detection rate than the benchmark algorithm. For example, our algorithm produced 25 false alarms and missed one detection and the benchmark algorithm produced 86 false alarms and missed 13 detections.

\section{CONCLUSION}

In this paper, we addressed a target detection problem for SAR images. We proposed a detection algorithm using a rotation invariant local descriptor. Our detection algorithm consists of rotation invariant local descriptor, supervised learning, feature weighting and diversity combining. The rotation invariant local descriptor makes our target detection algorithm robust against rotation of targets and more suitable for scenarios where target 


\begin{tabular}{|c|c|c|}
\hline Processing step & Parameter & Value \\
\hline Preprocessing & Averaging kernel size & $5 \times 5$ pixels \\
\cline { 2 - 3 } & Denoising threshold & 0.25 \\
\hline Rotation invariant transform & Discrete $\theta$ values & $0^{\circ}, 15^{\circ}, 30^{\circ}, 45^{\circ}, \ldots, 180^{\circ}$ \\
\hline Feature extraction & Sliding window size & $19 \times 19$ pixels \\
\hline \multirow{3}{*}{$\begin{array}{c}\text { I-RELIEF feature } \\
\text { weighting }\end{array}$} & Maximum number of iterations & 500 \\
\cline { 2 - 3 } & Distance metric & $f(d)=e x p(-d / \sigma)$ \\
\cline { 2 - 3 } & Kernel function & 25 \\
\cline { 2 - 3 } Classification & Kernel width $\sigma$ & 3.0 \\
\hline \multirow{3}{*}{ Post processing } & Threshold $\lambda$ on DR & 35 pixels \\
\hline Evaluation & Minimum number of & 10 pixel \\
\hline
\end{tabular}

Table 1: Parameters used to test performance

\begin{tabular}{|c|c|c|c|c|c|}
\hline \multicolumn{2}{|c|}{ Target image } & \multicolumn{2}{c|}{ Correct detections } & \multicolumn{2}{c|}{ False alarms } \\
\hline Mission & Pass & $\begin{array}{c}\text { Benchmark } \\
\text { Algorithm }\end{array}$ & $\begin{array}{c}\text { Our } \\
\text { Scheme }\end{array}$ & $\begin{array}{c}\text { Benchmark } \\
\text { Algorithm }\end{array}$ & $\begin{array}{c}\text { Our } \\
\text { Scheme }\end{array}$ \\
\hline 2 & 1 & 25 & 25 & 2 & 0 \\
\hline 3 & 1 & 22 & 25 & 1 & 4 \\
\hline 4 & 1 & 25 & 25 & 2 & 0 \\
\hline 5 & 1 & 23 & 25 & 4 & 2 \\
\hline 2 & 2 & 25 & 25 & 2 & 1 \\
\hline 3 & 2 & 25 & 25 & 4 & 1 \\
\hline 4 & 2 & 25 & 25 & 3 & 3 \\
\hline 5 & 2 & 25 & 25 & 4 & 2 \\
\hline 2 & 3 & 25 & 25 & 3 & 2 \\
\hline 3 & 3 & 23 & 25 & 4 & 1 \\
\hline 4 & 3 & 25 & 25 & 0 & 1 \\
\hline 5 & 3 & 24 & 25 & 2 & 0 \\
\hline 2 & 4 & 24 & 25 & 3 & 0 \\
\hline 3 & 4 & 25 & 25 & 2 & 0 \\
\hline 4 & 4 & 25 & 25 & 4 & 1 \\
\hline 5 & 4 & 25 & 24 & 4 & 1 \\
\hline 2 & 5 & 25 & 25 & 3 & 1 \\
\hline 3 & 5 & \multicolumn{5}{|c|}{$($ Used for training) } \\
\hline 4 & 5 & 25 & 25 & 2 & 2 \\
\hline 5 & 5 & 23 & 25 & 29 & 0 \\
\hline 2 & 6 & 25 & 25 & 1 & 1 \\
\hline 3 & 6 & 25 & 25 & 3 & 1 \\
\hline 4 & 6 & 25 & 25 & 1 & 1 \\
\hline 5 & 6 & 23 & 25 & 3 & 0 \\
\hline Total & 562 & 574 & 86 & 25 \\
\hline
\end{tabular}

Table 2: Comparing of results from benchmark algorithm and that from proposed scheme. Image of mission 3 pass 5 should not be counted in testing because it is used for training. 
orientation is significant. Feature weighting increases the discrimination between target and non-target classes. Compared with the baseline algorithm [1], our algorithm achieves fewer false alarms while achieving higher probability of target detection.

\section{Disclaimers}

The views and conclusions contained herein are those of the authors and should not be interpreted as necessarily representing the official policies or endorsements, either expressed or implied, of AFRL or the U.S. Government.

\section{Acknowledgement}

This material is based on research sponsored by AFRL under agreement number FA8650-06-1-1027. The U.S. Government is authorized to reproduce and distribute reprints for Governmental purposes notwithstanding any copyright notation thereon.

\section{REFERENCES}

1. M. Lundberg, L. Ulander, W. Pierson, and A. Gustavsson, "A challenge problem for detection of targets in foliage," Proceedings of SPIE , 2006.

2. L. Ulander, M. Lundberg, W. Pierson, and A. Gustavsson, "Change detection for low-frequency SAR ground surveillance," IEE Proceedings of Radar Sonar Navigation 152(6), pp. 413-420, December 2005.

3. "https://www.sdms.afrl.af .mil/datasets/vhf_change_detection/index.php."

4. Y. Sun, Z. Liu, S. Todorovic, and J. Li, "Adaptive boosting for synthetic aperture radar automatic target recognition," IEEE Transactions on Aerospace and Electronic Systems 43(1), pp. 112-125, January 2007.

5. L. Kaplan, "Improved SAR target detection via extended fractal features," IEEE Transactions on Aerospace and Electronic Systems 37(2), pp. 436-451, April 2001.

6. D. Howard, S. Roberts, and R. Brankin, "Target detection in SAR imagery by genetic programming," Advances in Engineering Software 30(5), pp. 303-311, May 1999.

7. T. Cooke, N. Redding, J. Schroeder, and J. Zhang, "Comparison of selected features for target detection in synthetic aperture radar imagery," Conference record of the 33rd Asilomar Conference on Signals, Systems, and Computers 2, pp. 859-863, 1999.

8. S. Kuttikkad and R. Chellappa, "Non-Gaussian CFAR techniques for target detection in high resolution SAR images," Proceedings of IEEE International Conference on Image Processing 1, pp. 910-914, 1994.

9. L. Ulander, P. Frolind, A. Gustavsson, H. Hellsten, and B. Larsson, "Detection of concealed ground targets in CARABAS SAR images using change detection," Proceedings of SPIE - International Society Optical Engineering 3721, pp. 243-252, 1999.

10. L. Ulander, B. Flood, P. Follo, P. Frolind, A. Gustavsson, T. Jonsson, B. Larsson, M. Lundberg, W. Pierson, and G. Stenstrom, "Flight Campaign Vidsel 2002, CARABAS-II change detection analysis," (FOI-R-1001SE), 2003.

11. L. Ulander, W. Pierson, M. Lundberg, and A. Gustavsson, "Performance of VHF-band SAR change detection for wide-area surveillance of concealed ground targets," Proceedings of SPIE - International Society Optical Engineering 5427, pp. 259-270, 2004.

12. L. Ulander, W. Pierson, M. Lundberg, P. Follo, P. Frolind, and A. Gustavsson, "CARABAS-II SAR change detection performance on ground targets concealed in foliage," Proceedings of EUSAR 2004, 5th European Conference on Synthetic Aperture Radar, Ulm, GE , pp. 297-300, 25-27 May 2004.

13. W. Ye, C. Paulson, D. Wu, and J. Li, "A target detection scheme for VHF SAR ground surveillance," Proceedings of SPIE Defense and Security Symposium 2008, Orlando, FL, 2008.

14. K. Mikolajczyk and C. Schmid, "A performance evaluation of local descriptors," IEEE Transactions on Pattern Analysis and Machine Intelligence 27(10), pp. 1615-1630, 2005.

15. D. Lowe, "Object recognition from local scale-invariant keypoints," Proceedings of the 7th International Conference on Computer Vision, pp. 1150-1157, 1999.

16. R. Bracewell, Two-Dimensional Imaging, Prentice Hall, Englewood Cliffs, NJ, 1995.

17. Y. Sun and J. Li, "Iterative RELIEF for feature weighting," Proceedings of the 23rd ACM International Conference on Machine learning 148, pp. 913-920, 2006. 